\title{
Three-Dimensional Elasticity Solutions for Isotropic and Generally Anisotropic Bodies
}

\author{
J.R.Barber \\ Department of Mechanical Engineering and Department of Civil and Environmental Engineering, \\ University of Michigan, Ann Arbor, MI 48109-2125, U.S.A. \\ jbarber@umich.edu
}

Keywords: Three-dimensional elasticity, Papkovich-Neuber solution, general anisotropy, Stroh formalism, prismatic bar

\begin{abstract}
Classical methods of two-dimensional elasticity can be extended to give an exact solution of the three-dimensional problem for the beam - i.e. a general solution for the prismatic bar loaded on its lateral surfaces, subject only to the restriction that the tractions can be expanded as power series in the axial coordinate $z$. A series of sub-problems $\mathcal{P}_{j}$ is defined by successive partial differentiations with respect to $z$. For isotropic materials, a recursive algorithm can be used for generating the solution to $\mathcal{P}_{j+1}$ from that for $\mathcal{P}_{j}$ in the context of the Papkovich-Neuber solution. For the generally anisotropic material, a similar strategy is proposed, based on partial integrations of Stroh's formulation of the two-dimensional problem.
\end{abstract}

\section{Introduction}

One of the major achievements in the theory of isotropic linear elasticity in the last century was the establishment of a general solution to the two-dimensional problem for the isotropic prismatic bar within the formalism of complex variable theory $[6,14,16,17]$. The problem naturally decomposes into two sub-problems: the plane strain problem [12], in which the stress and displacement fields are independent of distance $z$ along the axis of the bar and the only permissible tractions and body forces lie in the $x y$-plane, and the antiplane problem [13], in which the ends of the bar are subjected to prescribed force and/or moment resultants, but the lateral surfaces of the bar are traction-free. Certain stress and displacement components in the antiplane problem have low order polynomial dependence on $z$, but the solution reduces to that of a two-dimensional boundary value problem on the bar cross-section.

If the material is generally anisotropic the plane and antiplane problems become coupled. Alternative general solutions of the two-dimensional problem have been given by Stroh [18, 19] and Lekhnitskii [10]. Stroh shows that there exist three complex eigenvalue pairs $p$ such that a two-dimensional displacement field satisfying the equilibrium equations can be written in the form $\boldsymbol{u}=\boldsymbol{a} f(\zeta)$, where $\zeta=x+p y$ and $f$ is any function of $\zeta$. He constructs a general solution as a superposition of three such solutions, one for each eigenvalue. By contrast, Lekhnitskii starts by satisfying the equilibrium equations using the Airy and Prandtl stress functions. He then constructs the compatibility equations and shows that the resulting sixth order equation can be factorized into six first order equations. The solution again appears as the superposition of these separate equations in the form of functions of a single combination of $x, y$.

In principle, both Stroh and Lekhnitskii's methods permit a general solution of the twodimensional problem, though solution by complex variable methods is now complicated by the fact that different conformal mappings are required for each of the terms in the superposition. 


\section{Three-dimensional problems}

By contrast, there is no general solution for the three dimensional problem in elasticity. For the isotropic case, solutions of the equilibrium and compatibility equations can be written in terms of harmonic or biharmonic functions using the Papkovich-Neuber or Galerkin solution respectively [3]. This generally facilitates the solution of particular boundary-value problems, but in practice solutions are mostly restricted to a few simple geometries such as axisymmetric bodies, half-spaces and layers.

Even this device is not available for anisotropic materials unless the material has some convenient symmetry, but a few solutions have been obtained for the more general case, such as those for a concentrated point force in an infinite body or on the surface of a half-space. These are generally obtained using transform methods, such that the problem in the transform domain is two-dimensional and can therefore be treated using the Stroh or Lekhnitskii formalism $[20,22]$.

In this paper, we shall present some three-dimensional solutions for both isotropic and generally anisotropic materials. In particular, we shall present a general solution to the problem of the prismatic beam loaded on its lateral surfaces (i.e. not only on the ends). This can be considered as an exact three-dimensional theory of beams, in contrast to the Euler and Timoshenko theories, which are really low order approximations based on assumptions about the deformation of typical cross-sections. Problems of this class were first treated by Michell [11] and Almansi [1,2] for the cases where the loading was either uniform or linear in $z$. However, modern methods of solution, including the complex variable, Lekhnitskii and Stroh formalisms, greatly simplify the process of extending these methods to higher order variation with $z$.

\section{Superposition by differentiation}

Central to the method is the concept of generating new elasticity solutions by differentiating or integrating known solutions. If the displacement field $\boldsymbol{u}(x, y, z)$ satisfies the equations of elasticity for a homogeneous medium, so does $\boldsymbol{u}(x, y, z+a)$, since this represents the same field displaced a distance $a$ in the negative $z$-direction. Superposing the two fields and multiplying by the dimensionless constant $l / a$, we can construct the new solution

$$
\boldsymbol{u}^{\prime}=\frac{l(\boldsymbol{u}(x, y, z+a)-\boldsymbol{u}(x, y, z))}{a}
$$

which reduces to $l \partial \boldsymbol{u} / \partial z$ in the limit $a \rightarrow 0$. The length $l$ is introduced here just to retain dimensional consistency, but it can be set equal to unity without loss of generality.

This idea of developing new solutions by differentiation can be applied widely. For example, differentiating the point force 'Boussinesq' solution of Figure 1(a) with respect to $x$, we get the solution for a concentrated moment on the half space (Figure 1(b)).

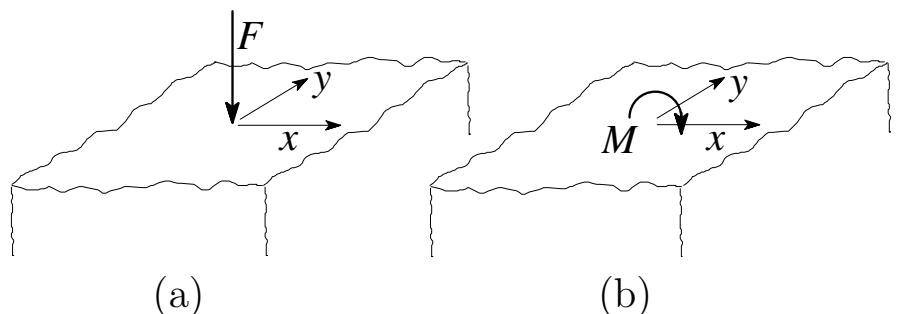

Fig. 1: Solution for a concentrated moment. 
As a second example, differentiating the Hertz solution of Figure 2 with respect to $x$, we obtain the solution for indentation by a tilted flat rigid elliptical punch, since the Hertz displacements are quadratic in $x, y$ in the elliptical contact area and their $x$-derivative is therefore a linear function of $x, y$ in this area, which defines a plane surface - i.e.

$$
\frac{\partial}{\partial x}\left(A x^{2}+B y^{2}+C\right)=2 A x
$$

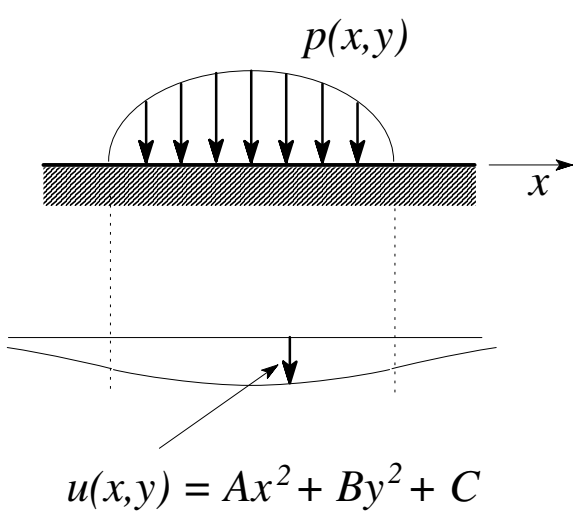

Fig. 2: The Hertzian pressure distribution.

Notice that sometimes the resulting solution is more complicated than that from which it is obtained. For example, a non-axisymmetric solution may be obtained from one that is axisymmetric.

The problem $\mathcal{P}_{m}$ Returning to the geometry of the prismatic bar, we consider the problem $\mathcal{P}_{m}$ defined such that the tractions on the lateral surfaces are

$$
T_{n}=\sum_{i=1}^{m-1} f_{i}(s) z^{i-1} ; \quad T_{t}=\sum_{i=1}^{m-1} g_{i}(s) z^{i-1} ; \quad T_{z}=\sum_{i=1}^{m} h_{i}(s) z^{i-1},
$$

where $\boldsymbol{n}, \boldsymbol{t}$ are the local outward normal and the tangent respectively to the closed curve(s) $\Gamma$ defining the boundary of the bar, and $f, g, h$ are arbitrary functions of the curvilinear coordinate $s$ defining position on $\Gamma$.

If the solution $\boldsymbol{u}^{(m)}$ of this problem is known, the new solution $\partial \boldsymbol{u}^{(m)} / \partial z$ will correspond to the tractions

$$
T_{n}=\sum_{i=1}^{m-1}(i-1) f_{i}(s) z^{i-2} ; \quad T_{t}=\sum_{i=1}^{m-1}(i-1) g_{i}(s) z^{i-2} ; \quad T_{z}=\sum_{i=1}^{m}(i-1) h_{i}(s) z^{i-2}
$$

and hence belongs to the class $\mathcal{P}_{m-1}$. Repeating this operation $m$ times, we establish a set of sub-problems $\mathcal{P}_{j}, j=0, m$ such that the solution

$$
\boldsymbol{u}^{(0)}=\frac{\partial^{m} \boldsymbol{u}^{(m)}}{\partial z^{m}}
$$

to the last in the series corresponds to the physical problem $\mathcal{P}_{0}$ in which the lateral tractions $\boldsymbol{T}=0$ and the only loading is on the ends. This is a classical antiplane problem problem that can in principle be solved for arbitrary cross-sections both for isotropic and anisotropic materials. In fact, since the stress field in the antiplane problem has at most linear dependence on $z$, two further differentiations with respect to $z$ yielda a displacement field equivalent to a null stress field and hence arbitrary rigid-body translation and rotation. 


\section{Partial integration}

In view of these results, it is clear that the original problem $\mathcal{P}_{m}$ could be solved if we could devise a method to generate the solution of $\mathcal{P}_{j+1}$ from that of $\mathcal{P}_{j}$. This process involves the determination of an appropriate partial integral of $\boldsymbol{u}^{(j)}$. This idea was first enunciated by Ieşan [8] and has since been applied by other authors in both analytical and numerical formulations $[5,9,15,4]$.

Integrating $\boldsymbol{u}^{(j)}$ with respect to $z$, we obtain

$$
\boldsymbol{u}^{(j+1)}=\int \boldsymbol{u}^{(j)} d z+\boldsymbol{f}(x, y),
$$

where $\boldsymbol{f}(x, y)$ is an arbitrary function of $x, y$ only which is required to satisfy two conditions:-

(i) The complete displacement field $\boldsymbol{u}^{(j+1)}$ including $\boldsymbol{f}(x, y)$ must satisfy the equations of elasticity in the strong sense, and

(ii) the stresses must reduce to the known tractions $\boldsymbol{T}^{(j+1)}$ on the lateral surfaces of the bar. Condition (ii) involves only the coefficient of $z^{0}$ in $\boldsymbol{T}^{(j+1)}$, since the coefficients of higher order terms will have been taken care of at an earlier stage in the recursive process. This condition can therefore be satisfied by superposing a corrective field that is the solution of a two-dimensional problem that can be solved by classical methods.

\section{Isotropic materials}

The conditions imposed by the equations of elasticity also only arise in the lowest order terms in the displacement field. If the material is isotropic, we can represent the displacements in terms of Papkovich-Neuber potentials [4] through the equations

$$
2 \mu u=2 \frac{\partial \phi}{\partial \bar{\zeta}}-(3-4 \nu) \psi+\zeta \frac{\partial \bar{\psi}}{\partial \bar{\zeta}}+\bar{\zeta} \frac{\partial \psi}{\partial \bar{\zeta}} ; \quad 2 \mu u_{z}=\frac{\partial \phi}{\partial z}+\frac{1}{2}\left(\bar{\zeta} \frac{\partial \psi}{\partial z}+\zeta \frac{\partial \bar{\psi}}{\partial z}\right)
$$

where $u=u_{x}+\imath u_{y}, \zeta=x+\imath y, \bar{\zeta}=x-\imath y$ and $\phi(\zeta, \bar{\zeta}, z), \psi(\zeta, \bar{\zeta}, z)$ are a real and a complex three-dimensional harmonic function respectively. In other words, the equilibrium condition will be satisfied by the stresses corresponding to (5) if and only if

$$
\frac{\partial^{2} \phi}{\partial z^{2}}+4 \frac{\partial^{2} \phi}{\partial \zeta \partial \bar{\zeta}}=0 ; \quad \frac{\partial^{2} \psi}{\partial z^{2}}+4 \frac{\partial^{2} \psi}{\partial \zeta \partial \bar{\zeta}}=0 .
$$

The complex variable form is particularly useful in the integration process (4). For example, if we have a function $\psi_{j}$ satisfying (6), we first integrate with respect to $z$, obtaining

$$
\psi_{j+1}=\psi_{j+1}^{P}+f(\zeta, \bar{\zeta})
$$

where

$$
\psi_{j+1}^{P}=\int \psi_{j}(\zeta, \bar{\zeta}, z) d z
$$

is any partial integral (not necessarily harmonic) and $f$ is an arbitrary function of $\zeta, \bar{\zeta}$. Substitution into (6) then yields

$$
4 \frac{\partial^{2} f}{\partial \zeta \partial \bar{\zeta}}=-4 \frac{\partial^{2} \psi_{j+1}^{P}}{\partial \zeta \partial \bar{\zeta}}-\frac{\partial^{2} \psi_{j+1}^{P}}{\partial z^{2}}
$$

The right hand side of this equation is necessarily independent of $z$, since the $z$-derivative of $\psi_{z+1}^{P}$ is $\psi_{j}$ from (8) and this satisfies (6) ex hyp. A suitable function $f$ can therefore always be obtained by integration of $(9)$ with respect to $\zeta$ and $\bar{\zeta}$. More detail of this procedure and an example is given in [4]. 


\section{Generally anisotropic materials}

The constitutive law for the generally anisotropic material takes the form

$$
\sigma_{i j}=C_{i j k l} \frac{\partial u_{k}}{\partial x_{l}}
$$

where the suffices are assumed to take the values $1,2,3$ and the stress components $\sigma_{i j}$ are required to satisfy the equilibrium equations

$$
\frac{\partial \sigma_{i j}}{\partial x_{j}}=C_{i j k l} \frac{\partial^{2} u_{k}}{\partial x_{j} \partial x_{l}}=0 .
$$

Suppose a particular displacement field $u_{k}^{[p]}\left(x_{1}, x_{2}, x_{3}\right)$ is known, which satisfies equation (11). We next construct a partial integral $u_{k}^{[p+1]}$ such that

$$
u_{k}^{[p]}=\frac{\partial u_{k}^{[p+1]}}{\partial x_{3}} .
$$

Since $u_{k}^{[p]}$ satisfies (11) ex hyp., we have

$$
C_{i j k l} \frac{\partial u_{k}^{[p+1]}}{x_{3} x_{j} x_{l}}=\frac{\partial}{\partial x_{3}}\left(C_{i j k l} \frac{\partial^{2} u_{k}^{[p+1]}}{\partial x_{j} \partial x_{l}}\right)=0
$$

and hence

$$
C_{i j k l} \frac{\partial^{2} u_{k}^{[p+1]}}{\partial x_{j} \partial x_{l}}=g\left(x_{1}, x_{2}\right),
$$

where $g\left(x_{1}, x_{2}\right)$ is a known function of $x_{1}, x_{2}$ only. This is identical to the equation of equilibrium for a body subjected to a known two-dimensional distribution of body force $p_{i}=-g\left(x_{1}, x_{2}\right)$. The development of a partial integral of $u_{k}^{[p]}$ that satisfies (11) is therefore reduced to a twodimensional body-force problem that could be solved, for example, using a suitable convolution on the known solution for a concentrated line force. However, we shall investigate more efficient methods of solution in the next section.

\section{The Stroh formalism}

Stroh $[18,19]$ investigated the conditions under which equations $(10,11)$ admit a solution of the form $u_{k}=\Re\left\{a_{k} f(\zeta)\right\}$, where $\zeta=x_{1}+p x_{2}$ and $p$ is a complex scalar parameter. Substituting into (11), we obtain the matrix equation

$$
\boldsymbol{D} \boldsymbol{a} \equiv\left\{\boldsymbol{Q}+p \boldsymbol{S}+p^{2} \boldsymbol{T}\right\} \boldsymbol{a}=0,
$$

where $Q_{i k}=C_{i 1 k 1}, S_{i k}=C_{i 1 k 2}+C_{i 2 k 1}, T_{i k}=C_{i 2 k 2}$. Equation (15) has a non-trivial solution if and only if the determinant

$$
\left|\boldsymbol{Q}+p \boldsymbol{S}+p^{2} \boldsymbol{T}\right|=0
$$

and this yields a sextic equation for $p$ which for physically realistic material properties always has three pairs of complex conjugate roots $p_{1}, p_{2}, p_{3}$. A general solution of the two-dimensional problem can then be constructed by superposition in the form

$$
\boldsymbol{u}=\Re\left\{\sum_{s=1}^{3} \boldsymbol{a} f_{s}\left(\zeta_{s}\right)\right\},
$$

where $\zeta_{s}=x_{1}+p_{s} x_{2}$. An elegant exposition of Stroh's formalism and its application to a wide range of problems is given by Ting [22]. 
Three-dimensional solutions The elegance of Stroh's solution and experience with the complex variable formulation of the isotropic problem suggests that three-dimensional solutions might best be sought as functions of $\left(\zeta, \bar{\zeta}, x_{3}\right)$, where $\bar{\zeta}=x_{1}+\bar{p} x_{2}$ is the complex conjugate of $\zeta$. We then have

$$
\frac{\partial}{\partial x_{1}}=\left(\frac{\partial}{\partial \zeta}+\frac{\partial}{\partial \bar{\zeta}}\right) ; \quad \frac{\partial}{\partial x_{2}}=\left(p \frac{\partial}{\partial \zeta}+\bar{p} \frac{\partial}{\partial \bar{\zeta}}\right)
$$

and the equilibrium equation can be written

$$
\mathcal{L}(\boldsymbol{u}) \equiv \boldsymbol{D} \frac{\partial^{2} \boldsymbol{u}}{\partial \zeta^{2}}+\frac{(\overline{\boldsymbol{U}}+\bar{p} \overline{\boldsymbol{V}})}{2} \frac{\partial^{2} \boldsymbol{u}}{\partial \bar{\zeta}^{2}}+(\boldsymbol{U}+\bar{p} \boldsymbol{V}) \frac{\partial^{2} \boldsymbol{u}}{\partial \zeta \partial \bar{\zeta}}+\boldsymbol{W} \frac{\partial^{2} \boldsymbol{u}}{\partial x_{3} \partial \zeta}+\overline{\boldsymbol{W}} \frac{\partial^{2} \boldsymbol{u}}{\partial x_{3} \partial \bar{\zeta}}+\boldsymbol{G} \frac{\partial^{2} \boldsymbol{u}}{\partial x_{3}{ }^{2}}=0
$$

where the overbar denotes the complex conjugate,

$$
\boldsymbol{U}=2 \boldsymbol{Q}+p \boldsymbol{S} ; \quad \boldsymbol{V}=\boldsymbol{S}+2 p \boldsymbol{T} ; \quad \boldsymbol{W}=\boldsymbol{E}+p \boldsymbol{F}
$$

and

$$
E_{i k}=C_{i 1 k 3}+C_{i 3 k 1} ; \quad F_{i k}=C_{i 2 k 3}+C_{i 3 k 2} ; \quad G_{i k}=C_{i 3 k 3} .
$$

Linear function of $x_{3}$ To gain some insight into the possible solutions of (19) that are polynomial in $x_{3}$, we first consider the simple (linear in $x_{3}$ ) trial function

$$
\boldsymbol{u}=\boldsymbol{a} x_{3} f_{1}(\zeta)+\boldsymbol{b} f_{2}(\zeta, \bar{\zeta})
$$

where $\boldsymbol{a}$ is the eigenfunction corresponding to a particular root $p$ of (16), $f_{1}$ is an arbitrary function of $\zeta$ only and $f_{2}$ is a function of both $\zeta$ and $\bar{\zeta}$. Since the first term is simply $x_{3}$ times Stroh's solution of the two-dimensional problem, we know that there will be no terms in $x_{3}$ after substitution in (19), so it should be possible to satisfy this condition by an appropriate choice of the additional two-dimensional function $f_{2}$. Substituting into (19), we obtain

$$
\mathcal{L}(\boldsymbol{u})=\boldsymbol{D} \boldsymbol{a} x_{3} f_{1}^{\prime \prime}(\zeta)+\boldsymbol{W} \boldsymbol{a} f_{1}^{\prime}+\boldsymbol{D} \boldsymbol{b} \frac{\partial^{2} f_{2}}{\partial \zeta^{2}}+\frac{(\overline{\boldsymbol{U}}+\bar{p} \overline{\boldsymbol{V}})}{2} \frac{\partial^{2} f_{2}}{\partial \bar{\zeta}^{2}}+(\boldsymbol{U}+\bar{p} \boldsymbol{V}) \frac{\partial^{2} f_{2}}{\partial \zeta \partial \bar{\zeta}} .
$$

The first term is zero in view of (15) and hence (19) will be satisfied if

$$
\boldsymbol{D} \boldsymbol{b} \frac{\partial^{2} f_{2}}{\partial \zeta^{2}}+\frac{(\overline{\boldsymbol{U}}+\bar{p} \overline{\boldsymbol{V}})}{2} \frac{\partial^{2} f_{2}}{\partial \bar{\zeta}^{2}}+(\boldsymbol{U}+\bar{p} \boldsymbol{V}) \frac{\partial^{2} f_{2}}{\partial \zeta \partial \bar{\zeta}}=-\boldsymbol{W} \boldsymbol{a} f_{1}^{\prime}(\zeta)
$$

At first sight, it appears that we can take $f_{2}$ to be an arbitrary function of $\zeta$ only and then solve the equation

$$
\boldsymbol{D} \boldsymbol{b} f_{2}^{\prime \prime}=-\boldsymbol{W} \boldsymbol{a} f_{1}^{\prime}(\zeta) .
$$

However, this strategy fails precisely because the matrix $\boldsymbol{D}$ is singular. The problem is similar to that which arises in other degenerate problems (see for example [3], §10.3). It can be resolved by the inclusion of an additional function $g(\zeta, \bar{\zeta})$ obtained from the 'obvious' form $f(\zeta)$ by differentiation with respect to the parameter (in this case $p$ ) whose special value causes the degeneracy. This differentiation yields the function

$$
g=\frac{\partial f(\zeta)}{\partial p}=f^{\prime}(\zeta) \frac{\partial \zeta}{\partial p}=x_{2} f^{\prime}(\zeta)
$$


However, in conformity with the notation introduced in (19), it is more convenient to write $x_{2}$ in terms of $\zeta, \bar{\zeta}$ and combine any arbitrary functions of the same variables, giving the form

$$
\boldsymbol{u}=\boldsymbol{a} x_{3} f_{1}(\zeta)+\boldsymbol{b} f_{2}(\zeta)+\boldsymbol{c} \bar{\zeta} f_{3}(\zeta)
$$

where $f_{1}, f_{2}, f_{3}$ are arbitrary functions of $\zeta$ only. Substitution into (19) and cancellation of any null terms resulting from (15) then yields

$$
\boldsymbol{D} \boldsymbol{b} f_{2}^{\prime \prime}+\boldsymbol{D} \boldsymbol{c} \bar{\zeta} f_{3}^{\prime \prime}+(\boldsymbol{U}+\bar{p} \boldsymbol{V}) \boldsymbol{c} f_{3}^{\prime}=-\boldsymbol{W} \boldsymbol{a} f_{1}^{\prime}(\zeta) .
$$

The term involving $\bar{\zeta}$ can be set to zero by choosing $\boldsymbol{c}=\boldsymbol{a}$, leaving

$$
\boldsymbol{D} \boldsymbol{b} f_{2}^{\prime \prime}+(\boldsymbol{U}+\bar{p} \boldsymbol{V}) \boldsymbol{a} f_{3}^{\prime}=-\boldsymbol{W} \boldsymbol{a} f_{1}{ }^{\prime} .
$$

A particular solution can then be found by writing

$$
f_{2}(\zeta)=\int f_{1}(\zeta) d \zeta ; \quad f_{3}(\zeta)=\gamma f_{1}(z)
$$

and choosing $\boldsymbol{b}$ to satisfy

$$
\boldsymbol{D} \boldsymbol{b}=-(\boldsymbol{W}+\gamma(\boldsymbol{U}+\bar{p} \boldsymbol{V})) \boldsymbol{a} .
$$

Since $\boldsymbol{D}$ is singular, (31) is solvable only if the right-hand side satisfies a certain condition. Premultiplying by $\boldsymbol{a}^{T}$ and noting that $\boldsymbol{a}^{T} \boldsymbol{D}=\mathbf{0}$, we have

$$
\boldsymbol{a}^{T}(\boldsymbol{W}+\gamma(\boldsymbol{U}+\bar{p} \boldsymbol{V})) \boldsymbol{a}=0
$$

and hence

$$
\gamma=-\frac{\boldsymbol{a}^{T} \boldsymbol{W} \boldsymbol{a}}{\boldsymbol{a}^{T}(\boldsymbol{U}+\bar{p} \boldsymbol{V}) \boldsymbol{a}} .
$$

If this condition is satisfied, (31) has a solution for $\boldsymbol{b}$ which is indeterminate to within an arbitrary multiplier of the eigenfunction $\boldsymbol{a}$.

Higher order terms The procedure described in the previous section can be applied successively to generate solutions with higher order polynomial dependence on $x_{3}$. At each stage, degeneracy of the matrix $\boldsymbol{D}$ demands additional functions with correspondingly higher order dependence on $\bar{\zeta}$. We therefore explore the possibility of a polynomial solution to (19) of the form

$$
\boldsymbol{u}_{n}=\sum_{j=1}^{n} \sum_{i=1}^{j} \frac{\boldsymbol{c}_{j}^{i} x_{3}^{(n-j)} \bar{\zeta}^{(i-1)} f_{n}^{[j-i]}(\zeta)}{(n-j) !(i-1) !}
$$

where the notation $f^{[*]}(\zeta)$ represents a set of partial integrals of $f(\zeta)$ defined by the recurrence relations

$$
f^{[s]}(\zeta)=\frac{\partial f^{[s+1]}}{\partial \zeta} ; \quad f^{[0]}=f(\zeta) .
$$

Substituting into (19) and equating coefficients of like powers of $x_{3}$ and $\bar{\zeta}$, we obtain the recurrence relation

$$
\boldsymbol{D} \boldsymbol{c}_{j}^{i}=-\frac{(\overline{\boldsymbol{U}}+\bar{p} \overline{\boldsymbol{V}})}{2} \boldsymbol{c}_{j}^{i+2}-(\boldsymbol{U}+\bar{p} \boldsymbol{V}) \boldsymbol{c}_{j}^{i+1}-\boldsymbol{W} \boldsymbol{c}_{j-1}^{i}-\overline{\boldsymbol{W}} \boldsymbol{c}_{j-1}^{i+1}-\boldsymbol{G} \boldsymbol{c}_{j-2}^{i},
$$

where the vectors $\boldsymbol{c}_{t}^{s}$ are assumed to be zero for $s<1$ or $t<s$. 
As in the case of equation (31), the singularity of the matrix $\boldsymbol{D}$ implies (i) that equation (36) can only be solved if the right hand side satisfies the condition

$$
\boldsymbol{a}^{T}\left(\frac{(\overline{\boldsymbol{U}}+\bar{p} \overline{\boldsymbol{V}})}{2} \boldsymbol{c}_{j}^{i+2}+(\boldsymbol{U}+\bar{p} \boldsymbol{V}) \boldsymbol{c}_{j}^{i+1}+\boldsymbol{W} \boldsymbol{c}_{j-1}^{i}+\overline{\boldsymbol{W}} \boldsymbol{c}_{j-1}^{i+1}+\boldsymbol{G} \boldsymbol{c}_{j-2}^{i}\right)=0 .
$$

and (ii) that the solution for $\boldsymbol{c}_{j}^{i}$ then contains an arbitrary multiplier of the eigenvector $\boldsymbol{a}$. In each case, the unknown multiplier introduced is determined from the solvability condition for the next higher vector coefficient.

This procedure is sufficient to determine the function $\boldsymbol{u}_{n}$ of equation (34) for arbitrary $n$, except for the unspecified function $f_{n}(\zeta)$. A general polynomial solution of order $m$ can then be constructed as

$$
\boldsymbol{u}=\sum_{n=1}^{m} \boldsymbol{u}_{n}=\sum_{n=1}^{m} \sum_{j=1}^{n} \sum_{i=1}^{j} \frac{\boldsymbol{c}_{j}^{i} x_{3}^{(n-j)} \bar{\zeta}^{(i-1)} f_{n}^{[j-i]}(\zeta)}{(n-j) !(i-1) !}
$$

which contains $m$ arbitrary functions $f_{n}(\zeta)$. Further superposition over the three Stroh eigenvalues $p_{1}, p_{2}, p_{3}$ then provides a solution sufficiently general to satisfy arbitrary polynomial boundary values on the lateral surfaces of the prismatic bar.

\section{Practical considerations}

As in all anisotropic problems, the matrix algebra involved in the solution will lead to rather complicated expressions even for comparatively simple boundary-value problems. In most cases, it is therefore probably more practical to perform the solution of the two-dimensional problem numerically, whilst retaining the polynomial dependence on $z$ [7]. This semi-analytical method has been extended by Taciroglu \& Lu [21] to the case of piezoelectric materials, for which there are significant new applications in the field of smart structures.

However, it is worth noting that the coefficients $\boldsymbol{c}_{j}^{i}$ in (38) depend on the material properties, but are independent of the particular boundary-value problem, and in principle, they can therefore be calculated once and for all as numerical values for a given anisotropic material. Furthermore, the recurrence relation involves the solution of the same matrix equation at all stages of the recursion, so the operation lends itself to automated computation, using either a symbolic program such as Maple or Mathematica, or a conventional computer program. Maple codes for the exact solution of anisotropic beam problems are given by Rand \& Rovenski [15], using an extension of the Lekhnitskii formalism.

In the Stroh formalism, the solution at each stage involves the determination of a scalar multiplier from a solvability criterion, followed by the solution of the matrix equation (36). This process is readily performed by representing the solution as an eigenfunction expansion. Once again, the parameters required for this purpose are the same for all geometries, so the eigenvalues and eigenfunctions of $\boldsymbol{D}$ need only be calculated once for a given material.

\section{Conclusions}

The method outlined here provides the analytical solution to any problem for the prismatic bar for which (i) the corresponding two-dimensional problem can be solved in general and (ii) the tractions on the lateral surfaces have polynomial dependence on $z$.

Problems of this class rapidly become algebraically tedious with increasing powers of $z$, though the basic operations are straightforward and repetitive. However, improvements in symbolic languages such as Mathematica and Maple bring calculations for realistic beam problems within reach. Alternatively, semi-analytic methods can be employed, where the two-dimensional problem is solved numerically for each power of $z$ in the polynomial expansion. 


\section{Acknowledgements}

The author wishes to thank Professor T.C.T.Ting for his significant contributions to the section on generally anisotropic materials.

\section{References}

[1] Almansi, E., "Sopra la Deformazione dei Cilinri Solecitati Lateralmente", Atti Real Accad. naz. Lincei Rend, Cl. sci fis., mat e natur. Ser. 510 (I), pp. 333-338, 1901a.

[2] Almansi, E., "Sopra la Deformazione dei Cilinri Solecitati Lateralmente", Atti Real Accad. naz. Lincei Rend, Cl. sci fis., mat e natur. Ser. 510 (II), pp. 400-408, 1901b.

[3] Barber, J. R., Elasticity, 2nd edn, Kluwer, Dordrecht, 2002.

[4] Barber, J. R., "Three-dimensional Elasticity Problems for the Prismatic Bar", Proc.Roy.Soc. (London), 462, pp. 1877-1896, 2006.

[5] El Fatmi, R. \& Zenzri, H., A Numerical Method for the Exact Elastic Beam Theory. Applications to Homogeneous and Composite Beams, Int.J.Solids Structures, 41, pp. 2521-2537, 2004.

[6] Green, A. E. \& Zerna, W., Theoretical Elasticity, Clarendon Press, Oxford, 1954.

[7] Huang, C.H. \& Dong, S.B., Analysis of Laminated Circular Cylinders of Materials with the most General Form of Cylindrical Anisotropy: I Axially Symmetric Deformations, Int.J.Solids Structures, 68, pp. 6163-6182, 2001.

[8] Ieşan, D., On Saint-Venant's Problem, Arch.Rational Mech.Anal., 91, pp. 363-373, 1986.

[9] Ladevèze, P., Sanchez, Ph., \& Simmonds, J.G., Beamlike (Saint-Venant) Solutions for Fully Anisotropic Elastic Tubes of Arbitrary Closed Cross Section, Int.J.Solids Structures, 41, pp. 1925-1944, 2004.

[10] Lekhnitskii, S. G., Theory of Elasticity of an Anisotropic Elastic Body, Holden-Day, San Francisco, 1963.

[11] Michell, J. H., "The Theory of Uniformly Loaded Beams", Quart. J. Math. 32, pp. 28-42, 1901.

[12] Milne-Thomson, L. M., Plane Elastic Systems, 2nd edn, Springer, Berlin, 1968.

[13] Milne-Thomson, L. M., Antiplane Elastic Systems, Springer, Berlin, 1962.

[14] Muskhelishvili, N. I., Some Basic Problems of the Mathematical Theory of Elasticity, P.Noordhoff, Groningen, 1963.

[15] Rand, O. \& Rovenski, V.Y., Analytical Methods in Anisotropic Elasticity with Symbolic Computational Tools, Birkhäuser, Boston, 2005.

[16] Stevenson, A. C., "Some Boundary Problems of Two-dimensional Elasticity", Phil. Mag., 34, pp. 766-793, 1943.

[17] Stevenson, A. C., "Complex Potentials in Two-dimensional Elasticity", Proc. Roy. Soc. (London)., 184A, pp. 129-179, 1945.

[18] Stroh, A. N., "Dislocations and Cracks in Anisotropic Elasticity", Phil.Mag., 3, pp. 625-646, 1958.

[19] Stroh, A. N., "Steady-state Problems in Anisotropic Elasticity", J.Math.Phys., 41, pp. 77-103, 1962. 
[20] Sveklo,V.A., "Boussinesq Type Problems for the Anisotropic Half-space", J.Appl.Math.Mech., 28, pp. 1099-1105, 1964.

[21] Taciroglu, E. \& Liu, C.W., Analysis and Design of Multimodal Piezoelectric Layered Tubular Sensors and Actuators, Smart Matls. Structures, 11, pp. 605-614, 2005.

[22] Ting, T. C. T., Anisotropic Elasticity, Oxford University Press, New York, 1996. 